\title{
Negative symptoms and longitudinal grey matter tissue loss in adolescents at risk of psychosis: preliminary findings from a 6-year follow-up study
}

Andrew G. McKechanie, Thomas W. J. Moorhead, Andrew C. Stanfield, Heather C. Whalley, Eve C. Johnstone, Stephen M. Lawrie and David G. C. Owens

\section{Background}

Negative symptoms are perhaps the most disabling feature of schizophrenia. Their pathogenesis remains poorly understood and it has been difficult to assess their development over time with imaging techniques.

\section{Aims}

To examine, using tensor-based structural imaging techniques, whether there are regions of progressive grey matter volume change associated with the development of negative symptoms.

\section{Method}

A total of 43 adolescents at risk of psychosis were examined using magnetic resonance imaging and whole brain tensorbased morphometry at two time points, 6 years apart.

\section{Results}

When comparing the individuals with significant negative symptoms with the remaining participants, we identified five regions of significant grey matter tissue loss over the 6-year period. These regions included the left temporal lobe, the left cerebellum, the left posterior cingulate and the left inferior parietal sulcus.

\section{Conclusions}

Negative symptoms are associated with longitudinal grey matter tissue loss. The regions identified include areas associated with psychotic symptoms more generally but also include regions uniquely associated with negative symptoms.

\section{Declaration of interest}

None.

\section{Copyright and usage}

(c) The Royal College of Psychiatrists 2016.
Schizophrenia remains a major cause of disability, with substantial personal and economic costs. Classically, the clinical features of schizophrenia are divided into positive symptoms and negative symptoms. Despite the clinical presentation being a mixture of both domains, it is the positive symptoms of the disorder which largely form the basis of the operationalised diagnostic criteria for schizophrenia, ${ }^{1,2}$ which show a response to antipsychotic medication ${ }^{3}$ and which have proven most tractable to research regarding their pathophysiology. ${ }^{4,5}$ However, the negative symptoms of schizophrenia are arguably more disabling ${ }^{6}$ and as yet there is no form of treatment which can be recommended as reliably relieving them. ${ }^{7}$ Advances in the development of new treatments for negative symptoms are hampered by a lack of understanding of their pathophysiology. A primary reason for this is that negative symptoms tend to be identified later rather than earlier in the course of schizophrenia ${ }^{8}$ making it unusual for them to be ratable in a situation uncontaminated by confounding factors. ${ }^{9}$ Principal among these confounders is antipsychotic medication - it has long been known that negative features may be difficult to distinguish from antipsychotic-induced akinesia. ${ }^{10}$ Other features associated with long-term psychotic illnesses, such as depression, ${ }^{11}$ institutionalisation ${ }^{12}$ and social isolation, ${ }^{13}$ also complicate the clinical picture in those with long-standing illness.

In the Department of Psychiatry in Edinburgh we have focused on the development of psychotic illness and as such have examined populations at enhanced risk of schizophrenia. First, we studied people where enhanced risk was familial, ${ }^{14}$ where we saw very little in terms of negative symptoms but this was not unexpected given the aims of the sample. We have gone on to examine individuals at enhanced risk for cognitive reasons ${ }^{15,16}$ and this is the sample used here. It is established that in people who are cognitively impaired, the risk of psychosis is higher, ${ }^{17}$ the onset earlier ${ }^{18}$ and that negative symptoms are more marked, ${ }^{19}$ meaning that the current sample is particularly suitable for their longitudinal investigation in the absence of the confounding factors associated with chronic illness. A number of cross-sectional studies have previously reported associations between negative symptoms in schizophrenia and tissue loss, in particular grey matter tissue loss bilaterally in the insula, superior temporal gyrus and amygdala/hippocampus; as well as the left superior temporal gyrus, medial temporal gyrus and occipital gyrus. ${ }^{20-22}$ Conversely, other studies have reported decreased tissue loss associated with negative symptoms. ${ }^{23,24}$ However, the assessment of negative symptoms in these studies is potentially confounded by the factors mentioned above. Therefore, the ability to assess negative symptoms repeatedly and in conjunction with serial scans in a substantial population, largely free from antipsychotic medication and at a much earlier age than in most other studies, allows us the unusual opportunity to examine the correlates of negative symptoms developing over time in this at-risk population.

\section{Method}

\section{Recruitment}

A full description of the recruitment and design of the Edinburgh Study of Comorbidity (from which this sample comes) is published elsewhere. ${ }^{15}$ In brief, local education authorities in Scotland were approached for permission to approach individual schools. Of the 19 authorities approached, 18 granted permission. A total of 243 schools across Scotland were subsequently approached and pupils receiving special educational assistance estimated to be functioning at a level consistent with borderline to mild intellectual disability were approached. After exclusions, 
394 participants were recruited to undergo further study. After screening the group with the Childhood Behaviour Checklist ${ }^{25}$ and the Structured Interview for Schizotypy, ${ }^{26}$ and sampling the group based on these scores to enrich the cohort for participants with higher scores on these measures, 168 were recruited for the baseline magnetic resonance imaging (MRI) assessment (mean age 15.7 years). Of these, 120 consented to be re-contacted for a follow-up scan 6 years later. At follow-up, we successfully re-contacted 105 families of whom 55 agreed to participate in our follow-up imaging.

\section{Clinical assessment}

The Clinical Interview Scale (CIS $)^{27,28}$ was used at each of the assessments, with the examinations being conducted by the same raters on each occasion. In addition, the participants were assessed on the Positive and Negative Syndrome Scale (PANSS) ${ }^{29}$ rated on the basis of this interview.

Using previously defined thresholds, ${ }^{16}$ the participants were divided into two groups using the scores in the negative symptoms subscale of the PANSS representing the presence or absence of morbid negative symptoms in this population. Individuals were allocated to the negative symptoms group if they had a score of 3 or above on any of the items of the negative subscale at either time-point, with the exception of concrete thinking and lack of spontaneity and flow of conversation. Most participants scored above a 1 on these items by virtue of their intellectual impairment, therefore we considered a score of 4 to represent definite morbidity. However, none of the participants was allocated to the negative symptoms group solely on the basis of their scores in either concrete thinking or lack of spontaneity and flow of conversation. All but six participants provided DNA samples to exclude diagnoses of Down syndrome, fragile-X syndrome or velocardiofacial syndrome.

\section{Image acquisition and pre-processing}

The MRI data were collected using a 1.5T GE Signa Horizon HDX (General Electric, Milwaukee, Wisconsin, USA). Following midline sagittal localisation, the whole brain was imaged by two further sequences: a transverse spin-echo scan acquiring both $\mathrm{T}_{2^{-}}$and proton density-weighted images; and a coronal gradient echo sequence acquiring 128 high resolution coronal $\mathrm{T}_{1}$-weighted images for structural image analysis (time of inversion $=600 \mathrm{~ms}$, echo time $=3.4 \mathrm{~ms}$, flip angle $=15^{\circ}$, field of view $=220 \mathrm{~mm}$, slice thickness $=1.7 \mathrm{~mm}$, matrix $=256 \times 192$ ).

The images were converted to ANALYZE 3D format and each image was examined for orientation and movement artefacts. The images were analysed in Statistical Parametric Mapping (SPM) version 5 (www.fil.ion.ucl.ac.uk) running in MATLAB version 7.3 (The MathWorks, Natick, Massachusetts, USA). The images were pre-processed using the SPM toolset before implementing the staged tensor-based morphometry (TBM) protocol ${ }^{30-33}$ to evaluate the grey matter tissue changes between the scans. For all scan acquisitions in this study we employed the same scanner, the same sequence and the same head coil. Nine participants were excluded from further analysis because of excess movement in the scans.

\section{TBM analysis}

The TBM protocol was implemented by following a staged procedure $^{31,32}$ and this was based on the established voxel-wise TBM methodology. ${ }^{30,33}$ The TBM method is susceptible to participant movement during scan time. As a result of this susceptibility, we were not able to use the paired scans from eight of the participants. Also, one participant did not complete the scan sequence. For each of the participants we recovered grey matter difference images in Montreal Neurological Institute (MNI) space from the TBM protocol.

The TBM grey matter difference images were smoothed with a $12-\mathrm{mm}$ full width half maximum filter and compared in SPM using the general linear model (GLM). Comparisons were made between those with threshold negative symptoms and those without such features. Age, inter-scan time interval, gender, full scale IQ and PANSS positive symptom score were included in the model as covariates. The SPM $t$-contrast was initially thresholded at $t=3.00$ (uncorrected) but we report results only where the cluster significance corrected for multiple comparisons was $P<0.05$. Removal of the covariates from the SPM cluster analysis increased the extent of the reported clusters and established that our analyses including covariates were conservative. The TBM-VBM whole-brain analysis was implemented in MNI standard space and the voxel coordinates of the results are presented in the same. The anatomical designations were taken from established atlases. ${ }^{34,35}$

Extracted values for whole brain grey matter volume were also compared to establish whether there was a relationship between change in volume and symptom states over time.

\section{Results}

\section{Description of participants}

Of the 55 participants scanned at baseline and 6-year follow-up, 46 had paired scans of sufficient quality to allow for the TBM analysis. Three further participants were excluded, as their lack of communication at baseline did not make it possible to make an assessment of negative symptomatology. Of these 43 participants, there were 13 females and 30 males, of mean age 15.6 years $($ s.d. $=1.8)$ at baseline and 21.6 years $($ s.d. $=1.8)$ at follow-up, and a mean IQ of 79.6. All participants either lived in their own home or in the family home at the time of the study. Of the participants, 5 were in further education, 4 in voluntary employment and 20 in paid employment, with the remainder being unemployed.

Table 1 shows the participants' characteristics as divided into the two clinical groupings. In general, the population was naive to psychotropic medication but two of them were on antipsychotic medication: one who had developed operationally defined schizophrenia since the beginning of the study and who was on depot flupentixol, lamotrigine and fluoxetine; the other participant was on small doses of amisulpiride and reboxetine for prominent anxiety symptoms. Both of these participants were in the negative symptoms group. A further two individuals, both in the negative symptoms-free group were taking antidepressant medication (sertraline and citalopram).

At baseline, the group as a whole had a mean PANSS negative subscale score of 10.6 (s.d.=2.6), and at follow-up a mean score of 11.8 (s.d.=4.6). Figure 1 shows the median PANSS negative subscale scores for the two groups at both baseline and 6-year follow-up. The negative symptom scores do not differ significantly at baseline $(P=0.069)$, although in the negative symptoms group are significantly higher at follow-up $(P<0.001)$. There was no significant difference in scores of observed depression on the CIS at baseline $(P=0.363)$.

At baseline, five participants met our threshold for negative feature pathology, of whom four had ongoing morbid negative symptoms at follow-up. In addition to these, a further four had developed threshold negative symptoms at 6-year follow-up.

\section{TBM analysis}

The whole brain TBM analysis found a number of clusters of significantly greater grey matter tissue loss between the scans at 


\begin{tabular}{|c|c|c|c|}
\hline & $\begin{array}{l}\text { Negative } \\
\text { symptoms-free } \\
\text { group }\end{array}$ & $\begin{array}{l}\text { Negative } \\
\text { symptoms } \\
\text { group }\end{array}$ & Significance \\
\hline$n$ (male: female) $^{\mathrm{a}}$ & $34(23: 11)$ & $9(7: 2)$ & 0.556 \\
\hline Age at baseline, ${ }^{b}$ years: mean (s.d.) & $15.2(1.3)$ & $17.2(2.4)$ & 0.009 \\
\hline Age at follow-up, ${ }^{\text {b years: mean (s.d.) }}$ & $21.1(1.3)$ & $23.4(2.4)$ & 0.003 \\
\hline Full-scale IQ, ${ }^{\mathrm{b}}$ mean (s.d.) & $80.8(15.8)$ & 75.0 (15.3) & 0.518 \\
\hline PANSS positive baseline, ${ }^{\mathrm{C}}$ median (IQR) & $8(2)$ & $9(5)$ & 0.163 \\
\hline PANSS negative baseline, ${ }^{\mathrm{C}}$ median (IQR) & $11(4)$ & $13(3)$ & 0.069 \\
\hline PANSS general baseline, ${ }^{\mathrm{C}}$ median (IQR) & $19.5(6)$ & $18(7)$ & 0.976 \\
\hline PANSS positive follow-up, ${ }^{\mathrm{C}}$ median (IQR) & $7.5(4)$ & $7(3)$ & 0.485 \\
\hline PANSS negative follow-up, ${ }^{\mathrm{C}}$ median (IQR) & $9.5(2)$ & $18(10)$ & $<0.001$ \\
\hline PANSS general follow-up, ${ }^{C}$ median (IQR) & $18(5)$ & $21(9)$ & 0.180 \\
\hline Baseline observed depression, ${ }^{c, d}$ median (IQR) & $0(0)$ & $0(1)$ & 0.363 \\
\hline Current alcohol units per week, median (IQR) & $2(10)$ & $1(3)$ & 0.141 \\
\hline Number who had ever used any illicit drugs ${ }^{\mathrm{e}}(\%)$ & $9(26)$ & $0(0)$ & \\
\hline Number currently using any illicit drug ${ }^{f}(\%)$ & $6(18)$ & $0(0)$ & \\
\hline \multicolumn{4}{|c|}{$\begin{array}{l}\text { PANSS, Positive and Negative Syndrome Scale; IQR, interquartile range. } \\
\text { a. } \chi^{2} \text { test. } \\
\text { b. Independent } t \text {-test. } \\
\text { c. Mann-Whitney U-test. } \\
\text { d. From the Clinical Interview Scale. } \\
\text { e. Nine individuals had previously used cannabis, four had used amphetamines, four had used ecstasy, three had used cocaine and one had used LSD. } \\
\text { f. Six individuals were currently using cannabis, one was currently also using mephedrone. }\end{array}$} \\
\hline
\end{tabular}

baseline and 6-year follow-up in those with negative symptoms, compared with those who had not. These clusters were in the occipital, inferior parietal and medial temporal lobes, as well as the posterior cingulate and the cerebellum. The significance levels for these clusters were calculated using the SPM non-stationary toolbox. Figure 2 shows the location and extent of these clusters. There were no regions of significantly greater tissue loss in the participants without negative symptoms when compared with the group with those symptoms. (Please note that a colour version of the figure is available in an online supplement to this paper.)

When the TBM analysis was repeated after excluding the two individuals on antipsychotic medication, the left medial temporal gyrus and left cerebellum results remained significant. However,



Fig. 1 Positive and Negative Syndrome Scale (PANSS) negative subscale scores at baseline and 6-year follow-up.

Error bars show $95 \%$ confidence interval. the other three results no longer remained significant. A third, new cluster in the cerebellum, however, did emerge as significant. The location of the maximal voxel for this cluster $(T=3.78)$ was at MNI coordinates $-6,-68,-38$.

\section{Whole brain grey matter volumes}

Figure 3 shows the results from our analysis of whole brain grey matter volumes over time. The differences between the groups are not significant at baseline $(P=0.881)$ or follow-up $(P=0.858)$, nor is the change over time $(P=0.591)$.

\section{Discussion}

In this study we undertook serial MRI brain scans of young individuals with intellectual limitations, scanning them at mean ages 16 and 22. Between these two time points, we found clusters of significantly greater grey matter tissue loss in the individuals with prominent negative symptoms compared with those without prominent negative symptoms. This included areas of greater tissue loss in the posterior cingulate, cerebellum, superior temporal gyrus, medial temporal lobe and occipital lobe all on the left-hand side. The groups did not differ significantly in terms of positive symptoms at either baseline or follow-up, nor were any of the participants admitted to hospital. The neuroanatomical changes over time that we report are therefore likely to relate to the presence of negative symptoms in this group and not to other confounding factors. One potential confounder is that although this group is almost completely antipsychotic naive, particularly when compared with other study populations, two of the individuals in the negative symptoms group were on antipsychotic medication. During further TBM analysis in which we excluded these individuals, two of the results remained significant and a new, significant result was found. We cannot know whether the loss of the significant results in the left superior temporal gyrus, left occipital lobe and left posterior cingulate is due to the absence of any antipsychotic medication effect or merely due to the smaller 
(a)

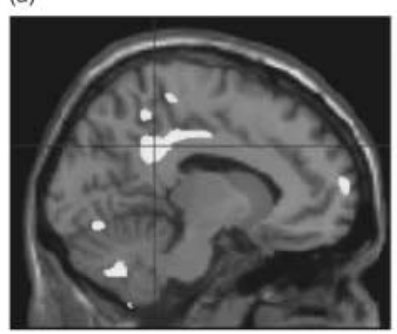

(b)

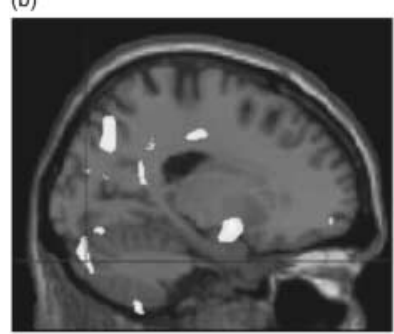

(c)

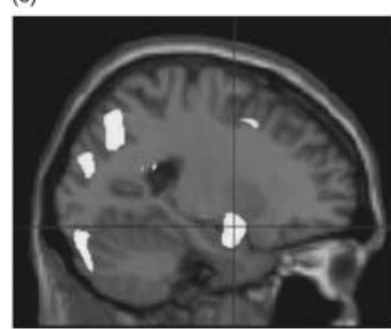

(d)

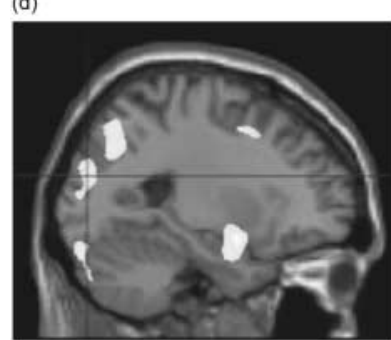

(e)
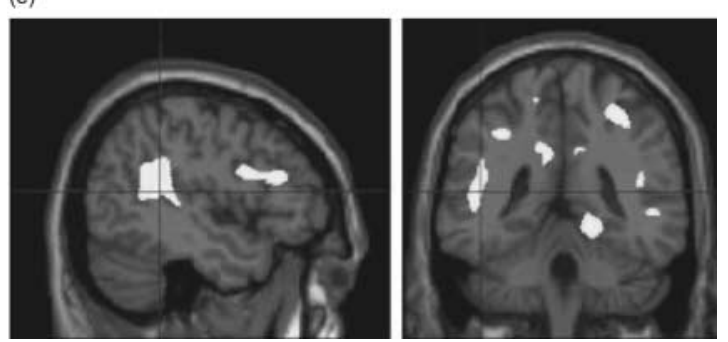

Fig. 2 (a) Cluster in the left posterior cingulate. The location of the maximal voxel for this cluster $(T=4.09)$ was at Montreal Neurological Institute (MNI) coordinates $-14,-48,29$ at a whole brain significance of $P<0.017$; (b) Cluster in the left cerebellum. The significance of this cluster at the whole brain level was $P<0.020$. The maximal voxel location for this cluster $(T=4.73)$ was at MNI coordinates $-18,-85,-31$; (c) Cluster including the left insula, amygdala and hippocampus. The location of the maximal voxel for this cluster $(T=4.86)$ was at MNI coordinates $-23,0,-14$ at a whole brain significance of $P<0.024$; (d) Cluster in the left occipatal lobe, extending forward to the left inferior parietal sulcus. The location of the maximal voxel $(T=3.92)$ was at MNI coordinates $-25,-83,21$. This cluster's significance at a whole brain level was $P<0.029$; (e) Cluster in the left superior temporal gyrus, extending to the supramarginalgyrus. The location of the maximal voxel for this cluster $(T=4.47)$ was at MNI coordinates $-46,-41,11$ at a whole brain significance of $P<0.037$.

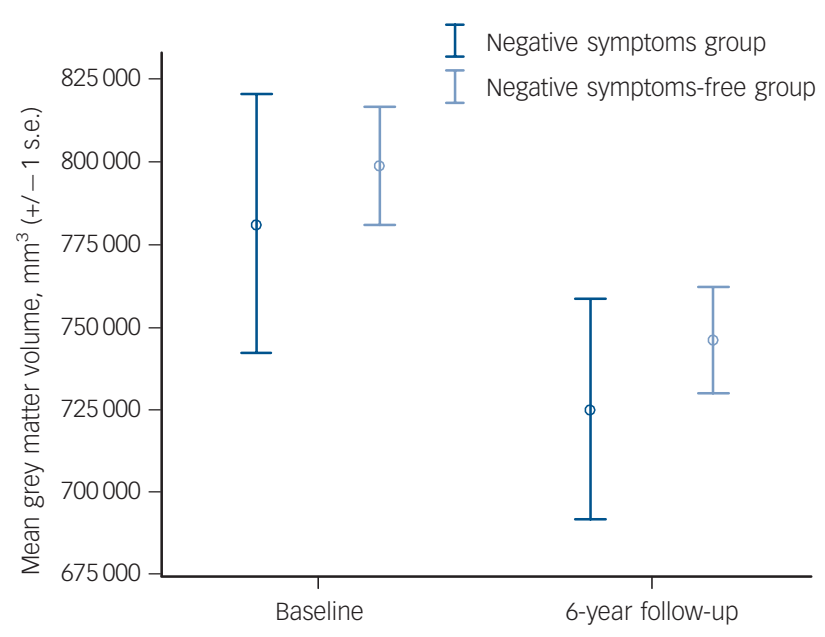

Fig. 3 Mean grey matter volumes at baseline and 6-year follow-up

sample size, but we can be sure that the development of negative symptoms in a sample uncontaminated by antipsychotic medications is associated with tissue loss in the left cerebellum and the left medial temporal gyrus.

To our knowledge, this is the first longitudinal study in a group at high risk of psychosis due to cognitive reasons to relate structural changes to increasing negative symptom severity. Within this group, we have previously demonstrated grey matter tissue loss in the bilateral medial temporal lobes and superior temporal gyri to be associated with threshold levels of positive and negative symptoms considered together ${ }^{16}$ (Table 2). The results of the current study overlap with these previous findings in relation to left medial temporal and superior temporal gyrus grey matter loss, but we also identified tissue loss in the left posterior cingulate, left cerebellum and left occipitoparietal region as uniquely associated with negative symptoms. Each of these regions has been previously reported to be associated with deficits in social cognition, which is known to be impaired in individuals with prominent negative symptoms. ${ }^{36}$

In the current study, we reported a large region of tissue loss at 6-year follow-up in the left posterior cingulate, which is consistent with the findings of a previous cross-sectional study of individuals with schizophrenia. ${ }^{37}$ It is possible that this finding may help the understanding of the nature of the specific deficits of negative

Table 2 Comparison of TBM correlates of negative features as reported in this study with the TBM findings in participants with all psychotic features as reported in Moorhead et $a^{16}$

$\begin{array}{ccc} & \text { All psychotic } & \text { Negative } \\ \text { Region of tissue loss } & \text { symptoms } & \text { symptoms }\end{array}$

Left temporal lobe
Superior temporal gyrus
Amygdala
Insula


symptoms. The posterior cingulate plays an important role in the default mode network ${ }^{38}$ and negative symptoms have been previously reported to be correlated with decreased connectivity between the posterior cingulate and various brain regions including frontal, temporal and midbrain structures ${ }^{39}$ as well as grey matter tissue loss in the posterior cingulate in individuals with schizotypal personality disorder. ${ }^{40}$ Theory of mind activity (the ability to attribute mental states to others) has also been linked to increased activity in the posterior cingulate ${ }^{41}$ and it is, therefore, of interest that we have found significant tissue loss in this area associated with prominent negative symptoms, which have been associated with deficits in theory of mind. ${ }^{42}$ Our finding suggests that this may be an area prone to tissue loss early in the course of a developing illness, and that tissue losses may be observable prior to the onset of illness and be involved in the aetiology of the disabling and treatment-refractory negative symptoms.

Our TBM results of tissue loss in the inferior parietal lobule are consistent with results from previous studies of negative symptoms in patients with schizophrenia ${ }^{43,44}$ and support the idea of the involvement of the inferior parietal lobule in the development of schizophrenia. This is in keeping with the hypothesised role of mirror neurons in the inferior parietal lobule with regard to social cognition, specifically empathy and understanding the actions and intentions of others. ${ }^{45,46}$ An alternative possible explanation is that it may relate to the importance of the parietal lobe in functions which are disturbed in schizophrenia such as spatial working memory, language and attention. ${ }^{47}$

Although more known for its role in motor coordination the cerebellum is increasingly recognised as having a variety of non-motor functions including a role in social cognition. ${ }^{48}$ Therefore, our finding of significant tissue loss in the left posterior cerebellum, which has previously been shown to be the region most associated with non-motor deficits, ${ }^{49}$ is in keeping with the evidence of its role in theory of mind as it relates to negative symptoms.

It is not clear to what degree prominent negative symptoms herald the development of schizophrenia, although it has more recently been suggested that negative symptoms may actually form an earlier, rather than later, part of the prodromal illness. ${ }^{50}$ However, when combined with the finding of Hazlett et al that decreased superior temporal gyrus volumes were found in individuals with schizophrenia compared with those with schizotypy, ${ }^{51}$ suggests that prominent negative symptoms and grey matter changes may indeed be the forbearer of a threshold illness.

\section{Study limitations}

There are certain limitations to this study. First, the group with negative symptoms is slightly but significantly older than those without. This is not unexpected as the older participants are closer to the peak age at onset of schizophrenia. However, as age was included as a covariate in the TBM analysis we know that the age difference does not explain our main results. Second, although it could be suggested that in the negative symptoms group we are merely looking at a group with more severe illness, and although in terms of negative symptoms this is clearly the case, it is not so in terms of positive symptoms which do not differ (Table 1). It is possible that the negative symptoms are a precursor to the development of manifest psychosis but this transition has yet to occur.

The number of individuals with negative symptoms is relatively small and confirmation in a larger sample would be desirable. However, the sample from which this group was derived was very large ${ }^{15,16}$ and the opportunity of observing the development of negative symptoms over time in a group as large as this is unusual. The significance of the negative symptoms in terms of their role as a precursor of the development of schizophrenia cannot be known at this stage. Finally, the inclusion of two individuals in the negative symptoms-free group who were taking antidepressants is a possible confounder, although among the group of 34, of whom 32 were medication free, we think it unlikely that this was driving the results. In addition, the measures of observed depression at baseline did not differ significantly between the groups.

\section{Implications}

The presence and development of negative symptoms in people at high risk of schizophrenia for cognitive reasons is related to grey matter tissue loss in brain regions associated with social cognition. Our findings compliment and add to those of previous studies in groups at increased risk for other reasons (e.g. genetic high risk or clinical high risk). As noted, the pathophysiology of negative symptoms has been poorly understood and effective treatments have been hard to find. The results presented here do not overcome these problems but may represent a first step towards meaningful understanding of the biological underpinnings of these disabling symptoms of schizophrenia.

\section{Andrew G. McKechanie, MRCPsych, The Patrick Wild Centre, The University of Edinburgh, Edinburgh; Thomas W. J. Moorhead, PhD, Division of Psychiatry, The University of Edinburgh, Edinburgh; Andrew C. Stanfield, PhD, MRCPsych, The University of Edinburgh, Edinburgh; Andrew C. Stanfield, PhD, MRCPSych, The
Patrick Wild Centre, The University of Edinburgh, Edinburgh; Heather C. Whalley, $P h D$, Eve C. Johnstone, MD, FRCP, FRCPsych, Stephen M. Lawrie, MD, FRCPE, FRCPsych, David G. C. Owens, MD, FRCP, FRCPsych, Division of Psychiatry,} The University of Edinburgh, Edinburgh, UK

Correspondence: Andrew G. McKechanie, The Patrick Wild Centre, The University of Edinburgh, Kennedy Tower, Tipperlinn Road, Edinburgh EH10 5HF, UK. Email address: andrew.mckechanie@ed.ac.uk

First received 11 Jul 2014, final revision 5 Oct 2014, accepted 20 Nov 2014

\section{Funding}

This study was supported by a programme grant from the UK Medical Research Council (G0100102) and by the Dr Mortimer and Theresa Sackler Foundation.

\section{Acknowledgements}

We are grateful to all of the study participants and their families, without whom this study would not have been possible. We are also most grateful to Dame Stephanie Shirley who funded the development of our mock scanning suite.

\section{References}

1 American Psychiatric Association. Diagnostic and Statistical Manual of Mental Disorders (5th edn). APA, 2013.

2 World Health Organization. International Statistical Classification of Disease and Related Health Problems, 10th Revision (ICD-10). WHO, 1992.

3 Davis JM, Schaffer CB, Killian GA, Kinard C, Chan C. Important issues in the drug treatment of schizophrenia. Schizophr Bull 1980; 6: 70-87.

4 Harrison PJ. The neuropathology of schizophrenia. A critical review of the data and their interpretation. Brain 1999; 122: 593-624.

5 Siever L, Davis KL. The pathophysiology of schizophrenia disorders: perspectives from the spectrum. Am J Psychiatry 2004; 161: 398-413.

6 Katschnig H. Schizophrenia and quality of life. Acta Psychiatr Scand Suppl 2000; 407: 33-7.

7 Hanson E, Healey K, Wolf D, Kohler C. Assessment of pharmacotherapy for negative symptoms of schizophrenia. Curr Psychiatry Rep 2010; 12: 563-71.

8 Pfohl B, Winokur G. The evolution of symptoms in institutionalized hebephrenic/catatonic schizophrenics. Br J Psychiatry 1982; 141: 567-72.

9 Carpenter Jr, WT, Heinrichs DW, Alphs LD. Treatment of negative symptoms. Schizophr Bull 1985; 11: 440-52. 
10 Quitkin F, Rifkin A, Klein DF. Very high dosage vs standard dosage fluphenazine in schizophrenia. A double-blind study of nonchronic treatment-refractory patients. Arch Gen Psychiatry 1975; 32: 1276-81.

11 Andreasen NC. Negative symptoms in schizophrenia. Definition and reliability. Arch Gen Psychiatry 1982; 39: 784-8.

12 Wing JK, Brown GW. Institutionalism and Schizophrenia: A Comparative Study of Three Mental Hospitals, 1960-1968. Cambridge University Press, 1970.

13 Harding CM, Brooks GW, Ashikaga T, Strauss JS, Breier A. The Vermont longitudinal study of persons with severe mental illness, I: methodology, study sample, and overall status 32 years later. Am J Psychiatry 1987; 144 718-26.

14 Johnstone EC, Ebmeier KP, Miller P, Owens DG, Lawrie SM. Predicting schizophrenia: findings from the Edinburgh High-Risk Study. Br J Psychiatry 2005; 186: $18-25$

15 Johnstone EC, Owens DG, Hoare P, Gaur S, Spencer MD, Harris J, et al. Schizotypal cognitions as a predictor of psychopathology in adolescents with mild intellectual impairment. Br J Psychiatry 2007; 191: 484-92.

16 Moorhead TWJ, Stanfield AC, MCKechanie AG, Dauvermann MR, Johnstone $\mathrm{EC}$, Lawrie SM, et al. Longitudinal gray matter change in young people who are at enhanced risk of schizophrenia due to intellectual impairment. Biol Psychiatry 2013; 73: 985-92.

17 Morgan VA, Leonard $\mathrm{H}$, Bourke J, Jablensky A. Intellectual disability co-occurring with schizophrenia and other psychiatric illness: population-based study. Br J Psychiatry. 2008; 193: 364-72.

18 Meadows G, Turner T, Campbell L, Lewis SW, Reveley MA, Murray RM Assessing schizophrenia in adults with mental retardation. A comparative study. Br J Psychiatry 1991; 158: 103-5.

19 Bouras N, Martin G, Leese M, Vanstraelen M, Holt G, Thomas C, et al. Schizophrenia-spectrum psychoses in people with and without intellectual disability. J Intellect Disabil Res 2004; 48: 548-55.

20 Anderson JE, Wible CG, McCarley RW, Jakab M, Kasai K, Shenton ME. An MRI study of temporal lobe abnormalities and negative symptoms in chronic schizophrenia. Schizophr Res 2002; 58: 123-34.

21 Wible CG, Anderson J, Shenton ME, Kricun A, Hirayasu Y, Tanaka S, et al. Prefrontal cortex, negative symptoms, and schizophrenia: an MRI study. Psychiatry Res 2001; 108: 65-78.

22 Cascella NG, Fieldstone SC, Rao VA, Pearlson GD, Sawa A, Schretlen DJ. Gray-matter abnormalities in deficit schizophrenia. Schizophr Res 2010; 120 63-70.

23 Galderisi S, Quarantelli M, Volpe U, Mucci A, Cassano GB, Invernizzi G, et al. Patterns of structural MRI abnormalities in deficit and nondeficit schizophrenia. Schizophr Bull 2008; 34: 393-401.

24 Volpe U, Mucci A, Quarantelli M, Galderisi S, Maj M. Dorsolateral prefrontal cortex volume in patients with deficit or nondeficit schizophrenia. Prog Neuropsychopharmacol Biol Psychiatry 2012; 37: 264-9.

25 Achenbach TM. Integrative Guide for the $1991 \mathrm{CBCL} / 4-18$, YSR, and TRF Profiles. University of Vermont Department of Psychiatry, 1991.

26 Kendler KS, Lieberman JA, Walsh D. The Structured Interview for Schizotypy (SIS): a preliminary report. Schizophr Bull 1989; 15: 559-71.

27 Goldberg DP, Cooper B, Eastwood MR, Kedward HB, Shepherd M. A standardized psychiatric interview for use in community surveys. Br J Prev Soc Med 1970; 24: 18-23.

28 Krawiecka M, Goldberg D, Vaughan M. A standardized psychiatric assessment scale for rating chronic psychotic patients. Acta Psychiatr Scand 1977; 55: 299-308.

29 Kay SR, Fiszbein A, Opler LA. The positive and negative syndrome scale (PANSS) for schizophrenia. Schizophr Bull 1987; 13: 261-76.

30 Kipps CM, Duggins AJ, Mahant N, Gomes L, Ashburner J, McCusker EA. Progression of structural neuropathology in preclinical Huntington's disease: a tensor based morphometry study. J Neurol Neurosurg Psychiatry 2005; 76 : 650-5.
31 Moorhead TW, McKirdy J, Sussmann JE, Hall J, Lawrie SM, Johnstone EC, et al Progressive gray matter loss in patients with bipolar disorder. Biol Psychiatry 2007; 62: 894-900.

32 Moorhead TW, Stanfield A, Spencer M, Hall J, McIntosh A, Owens DC, et al. Progressive temporal lobe grey matter loss in adolescents with schizotypal traits and mild intellectual impairment. Psychiatry Res 2009; 174: 105-9.

33 Whitford TJ, Grieve SM, Farrow TF, Gomes L, Brennan J, Harris AW, et al. Progressive grey matter atrophy over the first $2-3$ years of illness in first-episode schizophrenia: a tensor-based morphometry study. Neuroimage 2006; 32: 511-9.

34 Duvernoy HM. The Human Brain: Surface, Three-Dimensional Sectional Anatomy and MRI. Springer-Verlag, 1991.

35 Talairach J, Tournoux P. Co-Planar Stereotactic Atlas of the Human Brain. Thieme, 1988.

36 Pantelis C, Velakoulis D, McGorry PD, Wood SJ, Suckling J, Phillips LJ, et al. Neuroanatomical abnormalities before and after onset of psychosis: a cross-sectional and longitudinal MRI comparison. Lancet 2003; 361: 281-8.

37 Tanskanen P, Ridler K, Murray GK, Haapea M, Veijola JM, Jaaskelainen E, et al. Morphometric brain abnormalities in schizophrenia in a population-based sample: relationship to duration of illness. Schizophr Bull 2010; 36: 766-77.

38 Broyd SJ, Demanuele C, Debener S, Helps SK, James CJ, Sonuga-Barke EJ. Default-mode brain dysfunction in mental disorders: a systematic review. Neurosci Biobehav Rev 2009; 33: 279-96.

39 Bluhm RL, Miller J, Lanius RA, Osuch EA, Boksman K, Neufeld RW, et al. Spontaneous low-frequency fluctuations in the BOLD signal in schizophrenic patients: anomalies in the default network. Schizophr Bull 2007; 33: 1004-12.

40 Asami T, Whitford TJ, Bouix S, Dickey CC, Niznikiewicz M, Shenton ME, et al. Globally and locally reduced MRI gray matter volumes in neuroleptic-naive men with schizotypal personality disorder: association with negative symptoms. JAMA Psychiatry 2013; 70: 361-72.

41 Fletcher PC, Happe F, Frith U, Baker SC, Dolan RJ, Frackowiak RS, et al. Other minds in the brain: a functional imaging study of "theory of mind" in story comprehension. Cognition 1995; 57: 109-28.

42 Sprong $M$, Schothorst $P$, Vos $E$, Hox J, van Engeland $H$. Theory of mind in schizophrenia: meta-analysis. Br J Psychiatry 2007; 191: 5-13.

43 Sigmundsson $\mathrm{T}$, Suckling J, Maier M, Williams S, Bullmore E, Greenwood K, et al. Structural abnormalities in frontal, temporal, and limbic regions and interconnecting white matter tracts in schizophrenic patients with prominent negative symptoms. Am J Psychiatry 2001; 158: 234-43.

44 Frederikse M, Lu A, Aylward E, Barta P, Sharma T, Pearlson G. Sex differences in inferior parietal lobule volume in schizophrenia. Am J Psychiatry 2000; 157: 422-7.

45 Gallese V, Keysers C, Rizzolatti G. A unifying view of the basis of social cognition. Trends Cogn Sci 2004; 8: 396-403.

46 Rizzolatti G, Sinigaglia C. The functional role of the parieto-frontal mirror circuit: interpretations and misinterpretations. Nat Rev Neurosci 2010; 11: 264-74.

47 Choi JS, Park JY, Jung MH, Jang JH, Kang DH, Jung WH, et al. Phase-specific brain change of spatial working memory processing in genetic and ultra-high risk groups of schizophrenia. Schizophr Bull 2012; 38: 1189-99.

48 Calarge C, Andreasen NC, O'Leary DS. Visualizing how one brain understands another: a PET study of theory of mind. Am J PSychiatry 2003; 160: 1954-64.

49 Stoodley CJ, Schmahmann JD. Functional topography in the human cerebellum: a meta-analysis of neuroimaging studies. Neuroimage 2009; 44: 489-501.

50 Hafner $\mathrm{H}$, Maurer $\mathrm{K}$, an der Heiden W. ABC Schizophrenia study: an overview of results since 1996. Soc Psychiatry Psychiatr Epidemiol 2013; 48: 1021-31.

51 Hazlett EA, Buchsbaum MS, Haznedar MM, Newmark R, Goldstein KE, Zelmanova $Y$, et al. Cortical gray and white matter volume in unmedicated schizotypal and schizophrenia patients. Schizophr Res 2008; 101: 111-23. 